\title{
Fauna anura (Amphibia: Anura) asociada a jagüeyes en dos localidades de la región Caribe colombiana
}

\section{Anuran fauna (Amphibia: Anura) associated with farm ponds in two localities of the Colombian Caribbean region}

\author{
Cardozo, Jesús ${ }^{1 *}$ Biólogo, Caraballo, Pedro² Ph.D. \\ ${ }^{1}$ Universidad de Sucre. Grupo de Estudio en Zoología y Ecología - GEZEUS. Cra 28 N 5-265 \\ Barrio Puerta Roja. Sincelejo, Colombia. \\ ${ }^{2}$ Universidad de Sucre, Grupo de Investigación en Biodiversidad Tropical, \\ Línea de Ecología y Acuacultura Tropical. Sincelejo, Colombia.
}

\section{Keywords:}

Farm ponds; assemblages; complementarity.

\section{Palabras Clave:}

Jagüeyes;

ensamblaje;

complementariedad
INFORMACIÓN

Recibido: 01-11-2016;

Aceptado: 28-03-2017.

Correspondencia autor:

cardozojess@gmail.com

\section{Abstract}

The objective of the present study was to study the anurans species associated to ponds distributed among the municipalities of Galeras and Sincelejo. In the anuran composition, it was registered fifteen species from six families: Bufonidae (two), Ceratophrydae (one), Hylidae (six), Leptodactylidae (five) and Microhylidae (one). The richness and abundance presented variation for sampling and pond. The anuran fauna for these municipalities shares more than $93 \%$ of the species and it's characterized by species with wide distribution patterns, characteristic that could be related with modifications made to the environmental. The complementarity varied among $7.7-41.6 \%$ for the municipality of Sincelejo and 0-46.6\% for the municipality of Galeras. Studies like this they constitute to the ponds like habitat with implications to develop in both conservation and management strategies of species.

\section{Resumen}

Se estudió la fauna de anuros asociada a jagüeyes de los municipios de Galeras y Sincelejo. Se registró un total de quince especies de anuros agrupadas en cinco familias: Bufonidae (dos), Ceratophrydae (una), Hylidae (seis), Leptodactylidae (cinco) y Microhylidae (una). La riqueza y abundancia de especies varío entre los muestreos y jagüeyes. Los dos municipios estudiados comparten más del $93 \%$ de las especies, caracterizándose estos ensamblajes por un dominio de especies con amplios patrones de distribución, lo que es común en muchas zonas de bosque seco de Colombia. La complementariedad por pares de jagüeyes varió entre el 7.7-41.6\% para el municipio de Sincelejo y entre $0-46.6 \%$ para el municipio de Galeras. Estos resultados evidencian la importancia de los jagüeyes como hábitats para el desarrollo de estrategias que busquen el mantenimiento y conservación de especies. 


\section{INTRODUCCIÓN}

El agua dulce, que representa menos del $3 \%$ del agua disponible en la tierra, tiene una pequeña fracción epicontinental, de la cual los lagos son muy importantes, siendo cuerpos de agua, naturales y artificiales, con más de 2 ha de área (DOWNING et al., 2006) y los jagüeyes (conocidos como farm ponds) que son cuerpos de agua, naturales y artificiales, con áreas entre los $25 \mathrm{~m}^{2}$ y 2 ha, que pueden ser permanentes o estacionales (COLLINSON et al., 1995). En la costa Caribe colombiana los jagüeyes son cuerpos de agua artificiales y de tamaño variable, que están asociados con la producción agropecuaria y son conocidos también como pozos o represas, siendo construidos por remoción del suelo (excavación), represamiento en zonas de drenaje o una combinación de ambas formas. De estos cuerpos de agua depende una gran parte de la población en el Caribe para consumo o para el desarrollo de actividades agropecuarias.

Evidencias recientes muestran que estos pequeños cuerpos de agua tienen una contribución desproporcionadamente importante para la biodiversidad regional, en términos de riqueza y rareza de especies (BIGGS et al., 2007), lo que resalta su importancia ecosistémica, especialmente en zonas sin otros cuerpos de agua lénticos importantes, como la regiones Montes de María y Sabanas del departamento de Sucre. Como reservorios de diversidad, son importantes para la presencia de los ensambles de anuros, ya que sirven como sitios de reproducción, de alimentación e inclusive termo-regulatorios. Si bien los jagüeyes como cuerpos de agua juegan un papel fundamental como hábitats para los anuros, su presencia y distribución pude ser el resultado de la combinación de un complejo número de factores (FATORELLI y ROCHA, 2008). Siendo el hidroperíodo (ROGERS y CHALCRAFT, 2008) y la distribución de las lluvias (AICHINGER, 1987) unos de los de mayor importancia.
Factores relacionados con adaptaciones morfológicas, fisiológicas y/o filogenéticas, como los modos reproductivos, suelen influir en la utilización de un hábitat o microhábitat determinado (HADDAD y PRADO, 2005). En primer lugar, algunas especies de anuros necesitan cuerpos de agua para su reproducción lo que podría determinar su distribución espacial preferencialmente hacia este tipo de hábitats (CÁCERES-ANDRADE y URBINA-CARDONA, 2009) y en segundo lugar, características ecológicas propias de cada una de las especies le permiten colonizar hábitats que otras no pueden (VIEIRA et al., 2008). La transformación de los ecosistemas naturales en paisajes fragmentados a causa de las actividades antrópicas también influye en la composición de los ensamblajes de anuros, principalmente por la pérdida de una cobertura vegetal y cambios micro-climáticos (URBINAy LONDOÑO, 2003).

El departamento de Sucre cuenta con un registro de 38 especies de anuros (GALVÁN y DE LA OSSA, 2009; ACOSTA-GALVIS, 2012), sin embargo estos registros están limitados a muestreos rápidos de escasas localidades, dejando de lado una gran parte del territorio sucreño sin investigar. El presente trabajo fue desarrollado con el objetivo de evaluar la composición y similitud de los ensamblajes de anuros asociados a jagüeyes en dos municipios del departamento de Sucre.

\section{MATERIALES Y MÉTODOS}

Área de estudio. Este estudio fue desarrollado en los municipios de Sincelejo y Galeras, en el departamento de Sucre-Colombia (Fig. 1), ambos clasificados como pertenecientes al bosque seco tropical (HOLDRIDGE, 1967). Sincelejo presenta una temperatura promedio de $26.8^{\circ} \mathrm{C}$, una precipitación que varía entre 1.000 y 1.200 $\mathrm{mm} / \mathrm{año} \mathrm{y} \mathrm{una} \mathrm{humedad} \mathrm{relativa} \mathrm{del} 77 \%$. Para Galeras, la temperatura promedio de $27.2^{\circ} \mathrm{C}$, la precipitación fluctúa entre 990 y 1.275 mm/año y una humedad

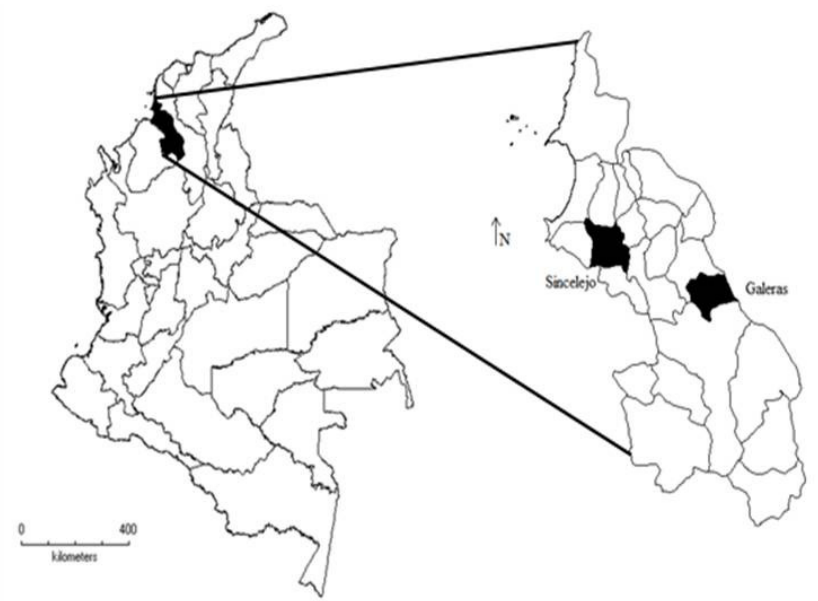

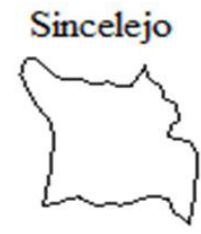

0

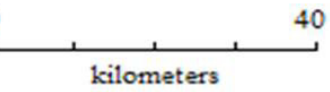

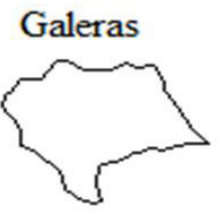

Figura 1. Ubicación de los municipios de Galeras y Sincelejo, Sucre. 
relativa del $80 \%$. La vegetación está compuesta por pocos residuos de vegetación secundaria, se dan rastrojos y extensas áreas de pastizales (AGUILERA, 2005).

Trabajo de campo. Se seleccionaron cinco jagüeyes por cada municipio teniendo en cuenta su accesibilidad y disponibilidad en las localidades estudiadas (Tabla 1). Todos los jagüeyes tienen residencia de agua permanente. Para los muestreos se estableció un área que fue delimitada mediante la suma no mayor de $30 \mathrm{~m}$ al radio medio de cada jagüey, que fue recorrido sólo una vez llevando un registro de todos los avistamientos y/o capturas.

Tabla 1. Localización y medida de los jagüeyes muestreados.

\begin{tabular}{cccc}
\hline Jagüey & Municipio & Área $\left(\mathrm{m}^{2}\right)$ & Coordenadas geográficas \\
\hline 1 & Sincelejo & 591 & $9^{\circ} 12^{\prime} 42.53^{\prime \prime} \mathrm{N} ; 75^{\circ} 24^{\prime} 05.12^{\prime \prime} \mathrm{O}$ \\
2 & Sincelejo & ---- & $9^{\circ} 14^{\prime} 49.55^{\prime \prime} \mathrm{N} ; 75^{\circ} 24^{\prime} 46.59^{\prime \prime} \mathrm{O}$ \\
3 & Sincelejo & 8.776 & $9^{\circ} 14^{\prime} 58.51^{\prime \prime} \mathrm{N} ; 75^{\circ} 24^{\prime} 44.77^{\prime \prime} \mathrm{O}$ \\
4 & Sincelejo & 1.632 & $9^{\circ} 13^{\prime} 57.63^{\prime \prime} \mathrm{N} ; 75^{\circ} 24^{\prime} 59.49^{\prime \prime} \mathrm{O}$ \\
5 & Sincelejo & 2.005 & $9^{\circ} 13^{\prime} 15.61^{\prime \prime} \mathrm{N} ; 75^{\circ} 24^{\prime} 29.87^{\prime \prime} \mathrm{O}$ \\
6 & Galeras & 2.403 & $9^{\circ} 11^{\prime} 42.27^{\prime \prime} \mathrm{N} ; 75^{\circ} 04^{\prime} 13.62^{\prime \prime} \mathrm{O}$ \\
7 & Galeras & 782 & $9^{\circ} 10^{\prime} 48.11^{\prime \prime} \mathrm{N} ; 75^{\circ} 03^{\prime} 40.79^{\prime \prime} \mathrm{O}$ \\
8 & Galeras & 497 & $9^{\circ} 10^{\prime} 28.68^{\prime \prime} \mathrm{N} ; 75^{\circ} 02^{\prime} 59.94^{\prime \prime} \mathrm{O}$ \\
9 & Galeras & 840 & $9^{\circ} 10^{\prime} 25.29^{\prime \prime} \mathrm{N} ; 75^{\circ} 03^{\prime} 20.92^{\prime \prime} \mathrm{O}$ \\
10 & Galeras & 420 & $9^{\circ} 09^{\prime} 56.89^{\prime \prime} \mathrm{N} ; 75^{\circ} 03^{\prime} 32.36^{\prime \prime} \mathrm{O}$ \\
\hline
\end{tabular}

Se efectuaron muestreos mensuales en cada jagüey, entre febrero y agosto del 2011, con el propósito de tener representatividad de las épocas de sequía (febrero a abril) y lluvias (mayo a agosto), empleando la técnica de muestreo por encuentro visual y captura manual (CRUMP y SCOTT, 1994) entre las 19:00-22:00 horas, para un total de 60 noches de muestreo. Los ejemplares se identificaron taxonómicamente en campo, y aquellos cuya identificación no fue posible fueron capturados y preservados siguiendo las metodologías descritas por ANGULO et al., (2006). Para luego ser comparados con material de referencia de la Sala de Colecciones Zoológicas de la Universidad de Córdoba (CZUC), en donde fueron depositados (la Universidad de Sucre y el Grupo de Investigación cuentan con el permiso para hacer este tipo de recolectas).

Análisis de datos. Se realizaron curvas de acumulación de especies para cada jagüey muestreado, para determinar la representatividad del muestreo, empleando los estimadores no paramétricos Jacknife de $1^{\text {er }}$ y $2^{\text {do }}$ orden (MORENO, 2001), con ayuda del programa EstimateS versión 8.2 (COLWELL, 2006). La similitud en la composición de especies por municipio fue verificada con la aplicación del índice de Jaccard, todo esto en el programa PAST 2.0 (HAMMER et al., 2001).
La riqueza de especies fue tomada como el número de especies avistadas y/o capturadas en cada sitio y la abundancia relativa como el número promedio de individuos contabilizados en cada una de las estaciones de muestreo, expresado este último en porcentaje. Se realizaron análisis de varianza (ANOVA) y posterior prueba de Tukey para comparar la variación de la riqueza y abundancia relativa por muestreo y jagüey, utilizando el software Statgraphics ${ }^{\circledR}$ centurión XVI previa transformación por logaritmo en base diez de los datos.

\section{RESULTADOS}

Se registraron 15 especies de anuros distribuidas en seis familias, lo cual corresponde al $39.47 \%$ de la riqueza específica de este grupo taxonómico para las áreas muestreadas en el departamento de Sucre. La familia con mayor riqueza de especies fue Hylidae, seguida de Leptodactylidae (Tabla 2). El jagüey número siete presentó la mayor riqueza, con 14 especies de anuros; aunque para el resto de los jagüeyes la riqueza osciló entre 9 y 13 especies (Tabla 2). En términos de composición de especies, Galeras y Sincelejo muestran una alta similitud compartiendo el $93.3 \%$ de las especies de anuros.

Tabla 2. Riqueza de especies de anuros registrados para los jagüeyes muestreados de los municipios de Galeras y Sincelejo, Sucre. Los números hacen referencia a la identificación de los jagüeyes.

\begin{tabular}{|c|c|c|c|c|c|c|c|c|c|c|c|}
\hline & \multirow{2}{*}{$\begin{array}{l}\text { Código } \\
\text { Especies }\end{array}$} & \multicolumn{10}{|c|}{ Jagüey } \\
\hline & & 1 & 2 & 3 & 4 & 5 & 6 & 7 & 8 & 9 & 10 \\
\hline \multicolumn{12}{|l|}{ Bufonidae } \\
\hline Rhinella humboldti & R.gra & $x$ & $x$ & $x$ & $x$ & $x$ & $x$ & $x$ & $x$ & & $x$ \\
\hline Rhinella marina & R.mar & $\mathrm{x}$ & $x$ & $x$ & $x$ & $x$ & $x$ & $x$ & $x$ & & $x$ \\
\hline \multicolumn{12}{|l|}{ Ceratophryidae } \\
\hline Ceratophrys calcarata & C.cal & $x$ & & & & & $x$ & $x$ & $x$ & & $x$ \\
\hline \multicolumn{12}{|l|}{ Hylidae } \\
\hline Dendropsophus microcephalus & D.mic & $x$ & $x$ & $x$ & $x$ & $x$ & $x$ & $x$ & $x$ & $x$ & $\mathrm{x}$ \\
\hline Hypsiboas crepitans & H.cre & $\mathrm{x}$ & $x$ & $x$ & $x$ & $x$ & $x$ & $x$ & $x$ & $x$ & $x$ \\
\hline Trachycephalus typhonius & T.ven & & & & & & & & & $\mathrm{x}$ & \\
\hline Pseudis paradoxa & Ps.pa & $x$ & $x$ & & $\mathrm{x}$ & $\mathrm{x}$ & & $x$ & $x$ & & $x$ \\
\hline Scinax rubra & S.rub & $\mathrm{x}$ & & & $x$ & $x$ & & $x$ & $x$ & & $x$ \\
\hline Scinax rostratus & S.ros & & $x$ & & & $x$ & & $x$ & & $\mathrm{x}$ & \\
\hline \multicolumn{12}{|l|}{ Leptodactylidae } \\
\hline Engystomops pustulosus & E.pus & $\mathrm{x}$ & $x$ & $x$ & & $\mathrm{x}$ & $\mathrm{x}$ & $x$ & $x$ & $x$ & $\mathrm{x}$ \\
\hline Pleurodema brachyops & P.bra & $\mathrm{x}$ & $x$ & $x$ & & $x$ & $x$ & $x$ & $x$ & $x$ & $\mathrm{x}$ \\
\hline Leptodactylus fuscus & L.fus & $\mathrm{x}$ & $x$ & $\mathrm{x}$ & $\mathrm{x}$ & $x$ & $\mathrm{x}$ & $x$ & $x$ & $\mathrm{x}$ & $\mathrm{x}$ \\
\hline Leptodactylus insularum & L.ins & $x$ & $x$ & $x$ & $\mathrm{x}$ & $x$ & $x$ & $\mathrm{x}$ & $\mathrm{x}$ & $\mathrm{x}$ & $\mathrm{x}$ \\
\hline Leptodactylus cf. fuscus & L.sp & $\mathrm{x}$ & $x$ & $\mathrm{x}$ & $x$ & $x$ & $x$ & $x$ & $x$ & $x$ & $x$ \\
\hline \multicolumn{12}{|l|}{ Microhylidae } \\
\hline Relictivomer paearsi & Re.pa & $x$ & $\mathrm{x}$ & & $\mathrm{X}$ & $\mathrm{x}$ & $x$ & $\mathrm{x}$ & $x$ & $\mathrm{x}$ & $\mathrm{x}$ \\
\hline Riqueza & & 13 & 12 & 9 & 10 & 13 & 11 & 14 & 13 & 10 & 13 \\
\hline
\end{tabular}


En cuanto a la abundancia relativa se registró un promedio de $71( \pm 19)$ individuos por jagüey durante las 60 noches de observación, siendo $D$. microcephalus la especie con mayor abundancia relativa en los dos municipios y el resto de las especies de anuros mostraron una abundancia relativa variable (Fig. 2).

Al realizar las comparaciones para los periodos establecidos (seco-lluvia) no se encontraron diferencias $(\mathrm{t}=0.6, \mathrm{P}=0.58)$, de allí que todas las comparaciones se realizaron utilizando el total de los muestreos.

Al comparar la variación de la riqueza se encontraron diferencias significativas por muestreo $\left(F_{[5,59]}=4.38, P=\right.$ $0.0025)$ y por jagüey $\left(F_{[9,59]}=10.13, P=0.000\right)$; al igual que para la abundancia relativa por muestreo $\left(F_{[5,59]}=\right.$ $17.21, P=0.000)$ y jagüey $\left(F_{[9,59]}=6.63, P=0.000\right)$. Los valores más bajos de riqueza se observaron durante los muestreos tres y cuatro, en donde la precipitación inicia su aumento (Fig. 3).

Las curvas de acumulación de especies observadas y estimadas (Fig. 4), reflejan que en términos generales se realizó un buen inventario de las especies de anuros asociadas a este tipo de hábitats, con una eficiencia en el muestreo que osciló entre el $88-97 \%$ para Galeras y $92-100 \%$, para Sincelejo. El estimador Jacknife de primer orden predice cerca de 16 especies, en tanto que Jacknife de segundo orden predice 17 especies. Las especies raras ("Singletons" y "Dubletons") se mantuvieron constantes en el transcurso de los

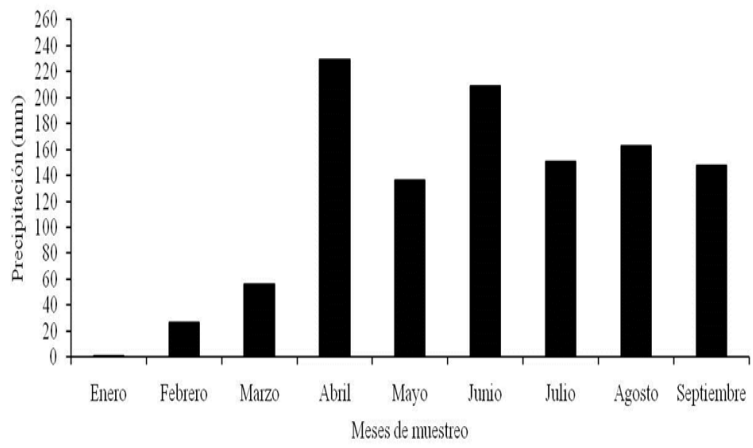

Figura 3. Valores de precipitación promedio mensual (mm) para el periodo de estudio. Datos suministrados por la estación meteorológica ubicada en la Universidad de Sucre, sede El Perico.

muestreos, con un leve incremento dado que $T$. typhonius fue vista una vez en los últimos eventos de muestreo para el municipio de Galeras.

El grado de recambio en la composición de especies (diversidad $\beta$ ) entre los jagüeyes estudiados fue bajo, pues la diferenciación en la composición del ensamblaje de anuros entre hábitats por municipio no varía considerablemente, compartiendo catorce de las quince especies registradas, con un valor de complementariedad entre el 7.7-41.6\% para Sincelejo y entre $0-46.6 \%$ para Galeras (Tabla 3 ). Estos valores muestran una alta similitud de los sitios estudiados.

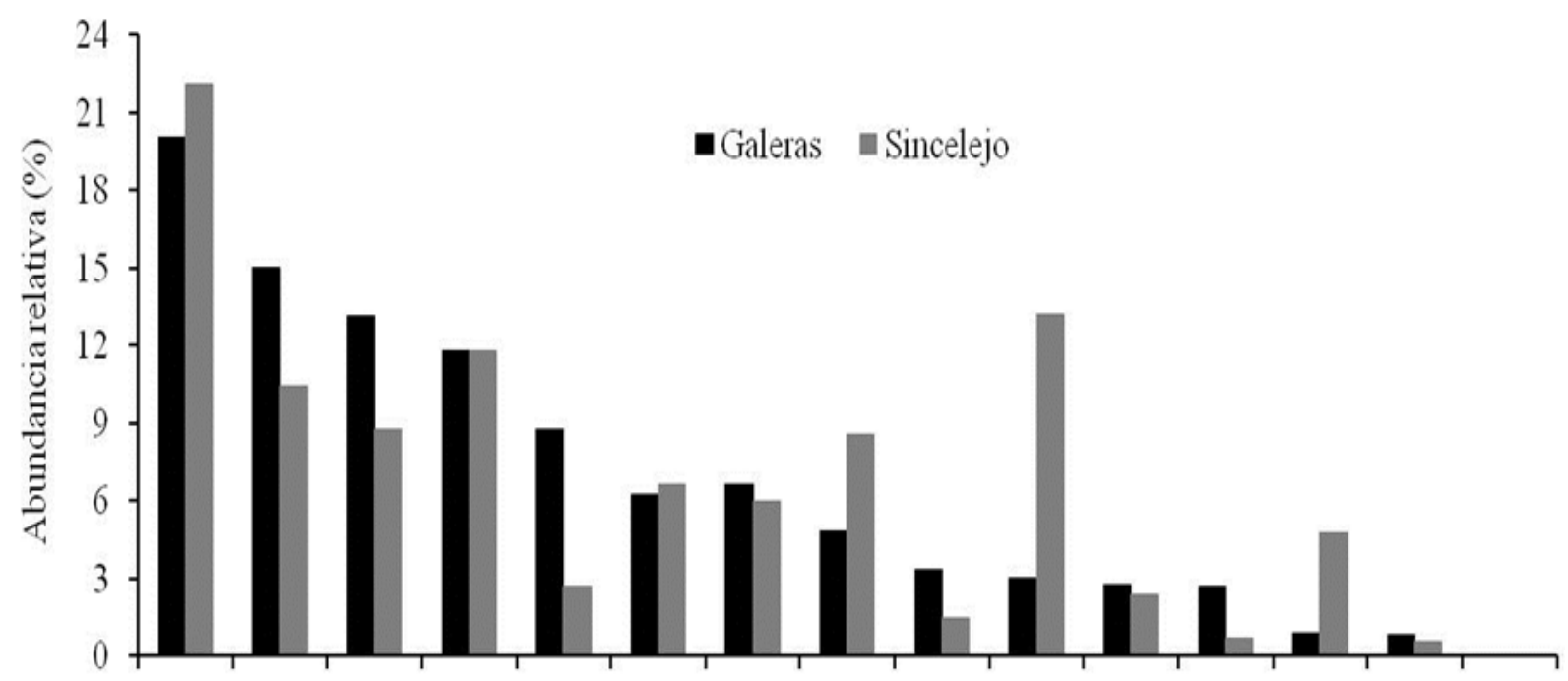

D.mic H.cre L.ins L.fus P.bra E.pus S.rub R.gra S.ros R.mar L.sp Re.pa Ps.pa C.cal T.ven Especies

Figura 2. Abundancia relativa de las especies de anuros para los municipios de Galeras y cada especie se encuentran registrados en la Tabla 2.

Sincelejo, Sucre. Los códigos de 


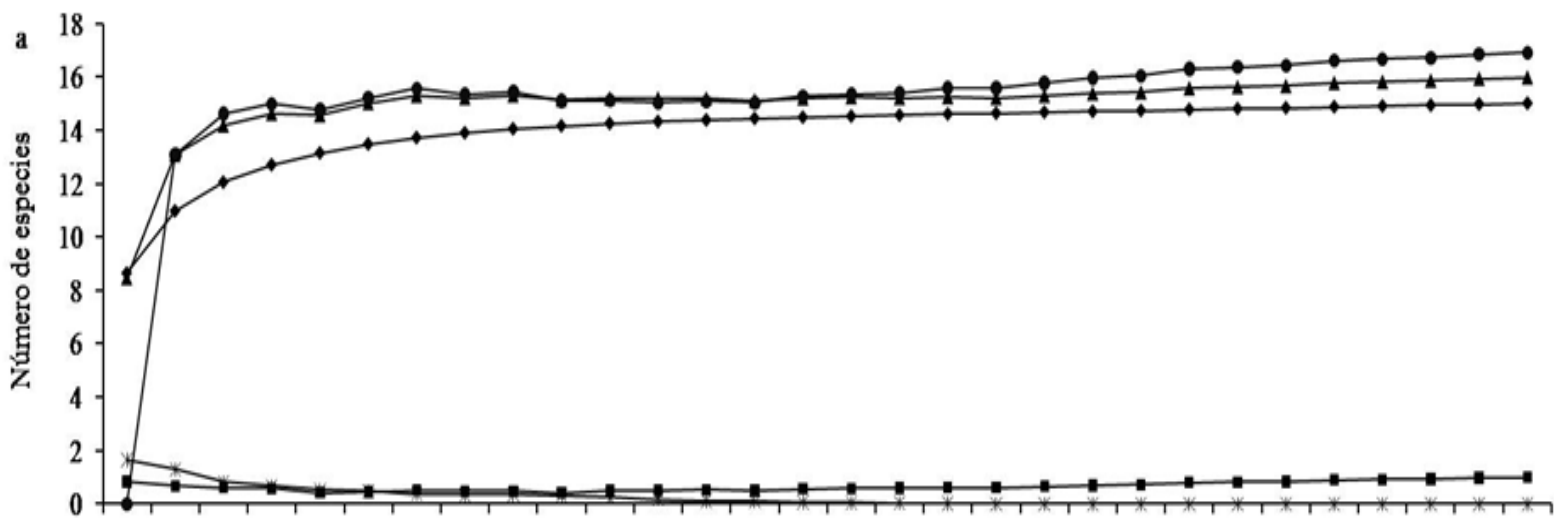

$\begin{array}{lllllllllllllllllllllllllllllll}1 & 2 & 3 & 4 & 5 & 6 & 7 & 8 & 9 & 10 & 11 & 12 & 13 & 14 & 15 & 16 & 17 & 18 & 19 & 20 & 21 & 22 & 23 & 24 & 25 & 26 & 27 & 28 & 29 & 30\end{array}$ Noches de muestreo

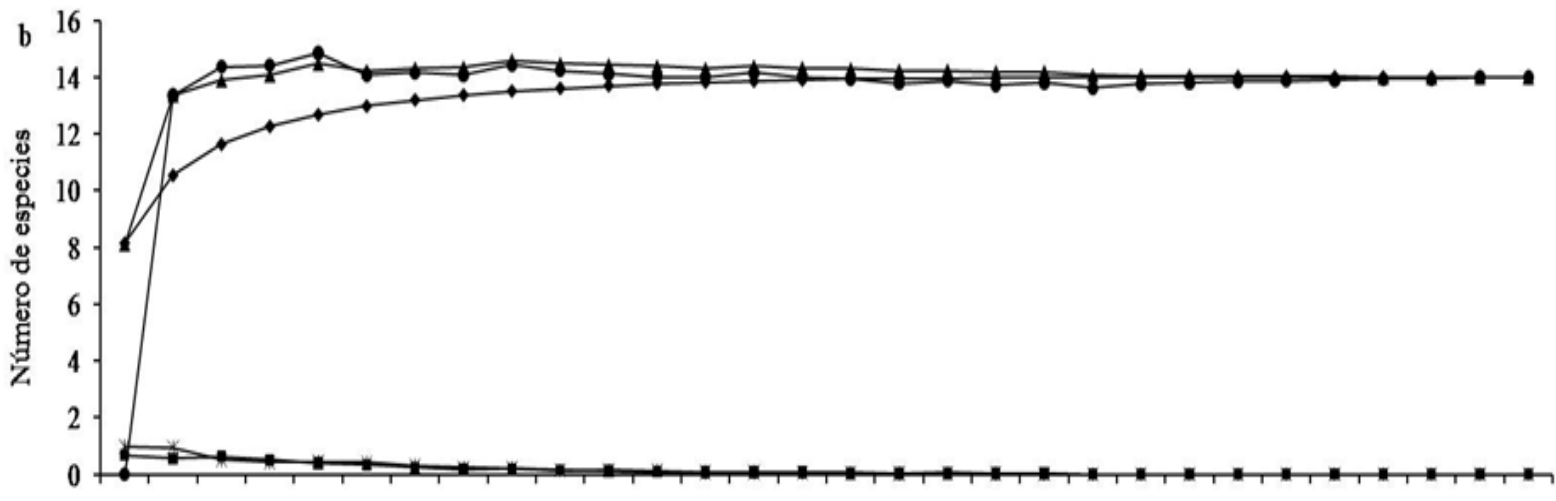

$\begin{array}{llllllllllllllllllllllllllllll}1 & 2 & 3 & 4 & 5 & 6 & 7 & 8 & 9 & 10 & 11 & 12 & 13 & 14 & 15 & 16 & 17 & 18 & 19 & 20 & 21 & 22 & 23 & 24 & 25 & 26 & 27 & 28 & 29 & 30\end{array}$

Noches de muestreo

Figura 4. Curva de acumulación de especies para los anuros de los municipios de Galeras (a) y Sincelejo (b). · = Jacknife 2 , $\boldsymbol{\Delta}$ $=$ Jacknife $1, "$ = Observado (Mao Tau), ${ }^{*}=$ Dobles, $\boldsymbol{\square}=$ Únicas.

Tabla 3. Valores porcentuales de similitud y complementariedad de las especies de anuros compartidas entre pares de jagüeyes para los municipios de Galeras y Sincelejo, Sucre. J = jagüey.

\begin{tabular}{|c|c|c|c|c|c|c|c|c|c|c|}
\hline Sincelejo & J1-J2 & J1-J3 & J1-J4 & J1-J5 & J2-J3 & J2-J4 & J2-J5 & J3-J4 & J3-J5 & J4-J5 \\
\hline $\mathrm{N}^{\circ}$ especies compartidas & 11 & 9 & 10 & 12 & 9 & 9 & 12 & 7 & 9 & 10 \\
\hline$N^{\circ}$ especies total entre pares de hábitats & 14 & 13 & 13 & 14 & 12 & 13 & 13 & 12 & 13 & 13 \\
\hline Porcentaje de similitud & 78.57 & 69.23 & 76.92 & 85.71 & 75 & 69.23 & 92.31 & 58.33 & 69.23 & 76.92 \\
\hline \multicolumn{11}{|l|}{ Complementariedad entre hábitats } \\
\hline Únicas & 3 & 4 & 3 & 2 & 3 & 4 & 1 & 5 & 4 & 3 \\
\hline Complementariedad & 0.21 & 0.31 & 0.23 & 0.14 & 0.25 & 0.31 & 0.077 & 0.416 & 0.31 & 0.23 \\
\hline Galeras & J6-J7 & J6-J8 & J6-J9 & J6-J10 & J7-J8 & J7-J9 & J7-J10 & J8-J9 & J8-10 & J9-10 \\
\hline $\mathrm{N}^{\circ}$ especies compartidas & 11 & 11 & 8 & 11 & 13 & 9 & 13 & 8 & 13 & 8 \\
\hline$N^{\circ}$ especies total entre pares de hábitats & 14 & 13 & 13 & 13 & 14 & 15 & 14 & 15 & 13 & 15 \\
\hline Porcentaje de similitud & 78.57 & 84.615 & 61.54 & 84.615 & 92.86 & 60 & 92.86 & 53.33 & 100 & 53.33 \\
\hline \multicolumn{11}{|l|}{ Complementariedad entre hábitats } \\
\hline Únicas & 3 & 2 & 5 & 2 & 1 & 6 & 1 & 7 & 0 & 7 \\
\hline Complementariedad & 0.21 & 0.15 & 0.38 & 0.15 & 0.071 & 0.4 & 0.071 & 0.466 & 0 & 0.466 \\
\hline
\end{tabular}




\section{DISCUSIÓN}

La fauna anura presente en los municipios de Galeras y Sincelejo, ya había sido reportada como de amplia distribución en Colombia y comunes en hábitats abiertos para las tierras bajas de Colombia (ACOSTA-GALVIS et al., 2006; LYNCH, 2006a). Las familias Hylidae y Leptodactylidae fueron las más representativas, patrón anteriormente reportado para áreas bajo la jurisdicción de la Corporación Autónoma Regional de Sucre (CARSUCRE) (GALVÁN y DE LA OSSA, 2009; ACOSTA-GALVIS, 2012), al igual que para otras regiones en el país (LYNCH et al., 1997; LYNCH, 2006a; ACOSTA-GALVIS et al., 2006).

La presencia de las especies aquí registradas podría estar relacionada con sus particularidades ecológicas que le permiten y/o facilitan ocupar estos hábitats. Según LYNCH, (1998) los hílidos tienen una alta diversidad en las tierras bajas, debido a que encuentran una gran cantidad de cuerpos de agua que son utilizados para su reproducción. Para miembros del género Leptodactylus, las áreas abiertas resultan ser sitios más apropiados para la construcción de nidos y para la vocalización de muchas especies (LUCAS et al., 2008) o por sus modos reproductivos, que al igual que para E. pustulosus consisten de nidos de espuma que reducen la exposición de las desovas a posibles depredadores (DOWNIE, 1990; 1993), esta adaptación también podría ser compartidas por los individuos de $P$. brachyops. Por lo que se refiere a $R$. marina y $R$. humboldtii, sus comportamientos termo-regulatorios relacionados con la selección de microhábitats húmedos (SEEBACHER y ALFORD, 1999), su amplio espectro en el consumo de presas (SAMPEDRO et al., 2011), asumir posturas relacionadas con minimizar la pérdida de agua por evapotranspiración (PRATES y NAVAS, 2009) y su toxicidad (HAYES et al., 2009), pueden ser características que le permiten favorecerse de los procesos de transformación de los ecosistemas.

La menor riqueza de especies de anuros se registró en los meses con mayores niveles de precipitación. Esta condición meteorológica permite que se formen charcas temporales, facilitando una mayor distribución horizontal de los sitios de reproducción y por esta razón una disminución de especies en el área muestreada de cada jagüey. Este comportamiento selectivo en la escogencia de estos sitios reproductivos (charcas temporales) ya había sido reportado (CÁCERESANDRADE y URBINA-CARDONA, 2009; Blanco y Bonilla, 2010) y pudiera deberse a las características de cada uno de estos sitios (por ejemplo, menor número de depredadores y competidores) y la ecología de las especies de anuros (ver DA SILVA y GIARETTA (2008) para una revisión de estudios de casos), lo que puede afectar la distribución de las especies que usan estos sitios para su reproducción. Este patrón ya fue establecido con anterioridad con los anfibios de la Ilanura Caribe (CUENTAS 1996, ROMERO \& LYNCH 2010, ROMERO \& LYNCH 2012).

Con relación a la abundancia relativa, factores como la pronunciada estación seca e imprevisibilidad en el inicio de las lluvias, pueden limitar la variedad de microhábitats húmedos necesarios para la reproducción de las especies de anuros (SANTOS et al., 2007). De allí que los jagüeyes sean hábitats de vital importancia para el desarrollo de los ensamblajes de anuros en estas localidades de la Región Caribe colombiana, no solo por presentar condiciones necesarias para la reproducción y sitios de alimentación, sino por proporcionar un hábitat con una cobertura vegetal y una mayor cantidad de microhábitats relativamente constantes a lo largo del año La distribución y preferencias de microhábitats por las especies de anuros pueden estar relacionadas con sus modos reproductivos (CÁCERES-ANDRADE y URBINA-CARDONA, 2009), ya que la reproducción de los anuros registrados en este estudio consiste en desovas directas en cuerpos de agua lénticos como los jagüeyes (LYNCH, 2006b). Más aún, si tenemos en cuenta características eco-fisiológicas de los anuros relacionadas con la preferencia por sitios húmedos, lo que podría estar relacionado con la distribución de las especies y el gran registro de las mismas. Estas características pueden hacer coincidir y/o concentrar la distribución de las especies de anuros en lugares relativamente pequeños en términos de área como los jagüeyes. Según ZIMMERMAN y BIERREGAARD, (1986) la disponibilidad de sitios reproductivos es uno de los factores de mayor importancia que determina la distribución de las especies de anuros.

La mayor abundancia de individuos se registró en los muestreos donde se observó un aumento en el volumen de precipitación, situación inversa para el caso de la riqueza. Esto puede ser explicado porque el incremento de las precipitaciones pudiera influenciar el suceso reproductivo en especies que depende de cuerpos de agua para su reproducción. Adicionalmente, durante este periodo fueron observados individuos de C. calcarata, P. brachyops, $P$ s. paradoxa e $H$. crepitans en amplexos y en últimos estadios de metamorfosis, lo que podría ser un indicio de actividad reproductiva de estas especies; sin embargo, son necesarios más estudios que evalúen este aspecto.

Para el caso de la baja complementariedad encontrada en este estudio podría estar relacionada con la estructura misma del paisaje, que en muchos aspectos es similar y eso genera un alto valor de similitud. Según VARGAS y BOLAÑOS, (1999a) la configuración del paisaje de las fincas dominadas por vegetación herbácea y pocos árboles dispersos permiten la coexistencia de muchas especies de anuros, pero dominados principalmente por especies de áreas abiertas. 
CONTE y ROSSA-FERES, (2006) evidencian la importancia de las formaciones morfoclimáticas en la distribución y composición de especies de anuros. De allí, que un factor no menos importante que podría explicar la presencia de especies con amplios patrones de distribución, es la topografía del departamento de Sucre, caracterizada por zonas de grandes planicies de sabanas y sin mayores elevaciones que generan una mayor homogeneidad ambiental, pues la diversidad de anuros en Colombia puede ser debida a la diversidad beta según lo sugerido por LYNCH et al., (1997). En términos generales este tipo de estudio permite resaltar la importancia de los jagüeyes como hábitats para los anuros y con potencialidad para el estudio de la ecología de las especies que los habitan.

\section{Agradecimientos}

Al grupo GEZEUS y Davi Teles por sus valiosos aportes y comentarios en el desarrollo de este manuscrito, a la profesora Ángela Ortega León por su colaboración en la facilitación de los especímenes de referencia de anuros de la CZUC, a los propietarios y trabajadores de las fincas en donde fue desarrollado este trabajo y a dos evaluadores anónimos por sus aportes.

\section{REFERENCIAS}

ACOSTA-GALVIS, A.R.; HUERTAS-SALGADO, C. \& RADA, M. 2006. Aproximación al conocimiento de los anfibios en una localidad del Magdalena medio (Departamento de Caldas, Colombia). Revista de la Academia Colombiana de Ciencias Exactas, Físicas y Naturales 30(115):291-303.

ACOSTA-GALVIS, A.R. 2012. Anfibios de los enclaves secos del área de influencia de los Montes de María y la Ciénaga de La Caimanera, departamento de Sucre, Colombia. Biota colombiana 13(2):211-231.

AGUILERA, M.M. 2005. La economía del departamento de Sucre: ganadería y sector público. Documento de trabajo sobre economía regional. Centro de Estudios Económicos Regionales. Banco de la República, Cartagena.

AICHINGER, M. 1987. Annual activity patterns of anurans in a seasonal neotropical environment. Oecologia 71(4):583-592.

ANGULO, A., RUEDA-ALMOACID, J.V.; Rodríguez-Mahecha, J.V. \& La Marca, E. (eds). 2006. Técnicas de Inventario y Monitoreo para los Anfibios de la Región Tropical Andina. Bogotá.

BIGGS, J.; WILLIAMS, P.; WHITEFIELD, M.; NICOLET, P.; BROWN, C.; HOLLIS, J.; ARNOLD, D. \& PEPPER, T. 2007. The freshwater biota of British agricultural landscapes and their sensitivity to pesticides. Agriculture, Ecosystems and Environment 122(2):137-148.

BLANCO TORRES, A. \& BONILLA GÓMES, M.A. 2010. Partición de microhábitats entre especies de Bufonidae y Leiuperidae (Amphibia: Anura) en áreas con bosque seco tropical de la región Caribe-Colombia. Acta Biológica Colombiana 15(3):47-60.

CÁCERES-ANDRADE, S. \& URBINA-CARDONA, J.N. 2009. Ensamblajes de anuros de sistemas productivos y bosques en el piedemonte llanero, departamento del Meta, Colombia. Caldasia 31(1):175-194.

COLLINSON, N.; BIGGS, J.; CORFIELD, A.; HODSON, M.J.; WALKER, D.; WHITFIELD, M. \& WILLIAMS, P.J. 1995. Temporary and permanent ponds: an assessment of the effects of drying out on the conservation value of aquatic macroinvertebrate communities. Biological Conservation 74(2):25-134.

COLWELL, R.K. 2006. EstimateS: statistical estimation of species richness and shared species from samples. Versión 8.2. Disponible en: http://viceroy.eeb.uconn.edu/estimates

CONTE, C. \& ROSSA-FERES, D. 2006. Diversidade e ocorrência temporal da anurofauna (Amphibia, Anura) em São José dos Pinhais, Paraná, Brasil. Revista Brasileira de Zoologia 23(1):162-175.

CRUMP, M. \& SCOTT, N. 1994. Visual Encounter Surveys:84-92. En: Heyer, R.; Donnelly, M. McDiarmid, R.; Hayek, L. \& Foster, M. (eds), Measuring and Monitoring Biological Diversity: standard methods for amphibians. Smithsonian Institution Press. Washington. 
DA SILVA, W. \& GIARETTA, A.A. 2008. Seleção de sítios de oviposição em anuros (Lissamphibia). Biota Neotropica 8:243-248.

DOWNIE, J.R. 1990. Functions of the foam in foam-nesting leptodactylids: anti-predator effects of Physalaemus pustulosus foam. Herpetological Journal 1(11):501-503.

DOWNIE, J.R. 1993. Functions of the foam in foam-nesting leptodactylids: the nest as a post-hatching refuge in Physalaemus pustulosus. Herpetological Journal 3(1):35-42.

DOWNING, J.A.; PRAIRIE, Y.T.; COLE, J.J.; DUARTE, C.M.; TRANVIK, L.J.; STRIEGL, R.G.; MCDOWELL, W.H.; KORTELAINEN, P.; CARACO, N.F.; MELACK, J.M. \& MIDDELBURG, J.J. 2006. The global abundance and size distribution of lakes, ponds, and impoundments. Association for the Sciences of Limnology and Oceanography 51(5):2388-2397.

FATORELLI, P. \& ROCHA, C.F. 2008. O que molda a distribuição das guildas de girinos tropicais? Quarenta anos de busca por padrões. Oecologia Brasil 12(4):733-742.

GALVÁN-GUEVARA, S. \& DE LA OSSA, J. 2009. Herpetofauna registrada para el área de influencia de la reserva forestal protectora Serranía de Coraza, Colosó, Sucre, Colombia. Revista colombiana de ciencia animal 1(2):250-258.

HADDAD, C. \& PRADO, C. 2005. Reproductive modes in frogs and their unexpected diversity in the Atlantic Forest of Brazil. BioScience 55(3):207-217.

HAMMER, Ø.; HARPER, D.A.T. \& RYAN, P.D. 2001. PAST: Paleontological Statistics Software Package for Education and Data Analysis. Palaeontologia Electronica 4(9):1-92

HAYES, R.A.; CROSSLAND, M.R.; HAGMAN, M.; CAPON, R.J. \& SHINE, R. 2009. Ontogenetic variation in the chemical defenses of Cane toads (Bufo marinus): toxin profiles and effects on predator. Journal of chemical ecology 35(4):391-399.

HOLDRIDGE, L. 1967. Life zone ecology. Tropical Science Center. San José de Costa Rica.

LUCAS, E.M.; BRASILEIRO, C.A.; OYAMAGUCHI, H.M. \& MARTINS, M. 2008. The reproductive ecology of Leptodactylus fuscus (Anura, Leptodactylidae): new data from natural temporary ponds in the Brazilian Cerrado and a review throughout its distribution. Journal of Natural History 42(35-36):2305-2320.

LYNCH, J.D. 1998. La riqueza de la fauna anfibia de los Andes colombianos. Innovación y Ciencia 7(4):46-51.

LYNCH, J.D. 2006a. The amphibian fauna in the Villavicencio Region of eastern Colombia. Caldasia 28(1):135-155.

LYNCH, J.D. 2006b. The tadpoles of frogs and toads found in the lowlands of northern Colombia. Revista de la Academia Colombiana de Ciencias Exactas, Físicas y Naturales 30(116):443-457.

LYNCH, J.D.; RUIZ-CARRANZA, P. \& ARDILA-ROBAYO, M.C. 1997. Biogeographic Paterns of Colombian Frogs and Toads. Revista de la Academia Colombiana de Ciencias Exactas, Físicas y Naturales 21(80):237-248.

MORENO, C.E. 2001. Métodos para medir la biodiversidad. Manuales \& Tesis SEA. Zaragoza, España.

PRATES, I. \& NAVAS, C.A. 2009. Cutaneous resistance to evaporative water loss in brazilian Rhinella (Anura: Bufonidae) from contrasting environments. Copeia 2009(3):618-622.

ROGERS, T.N. \& CHALCRAFT, D.R. 2008. Pond hydroperiod alters the effect of density-dependent processes on larval anurans. Canadian Journal of Fisheries \& Aquatic Sciences 65(12):2761-2768.

SAMPEDRO-MARÍN, A.C.; ANGULO, Y.Y.; ARRIETA, F.I. \& DOMÍNGUEZ, D.M. 2011. Alimentación de Bufo marinus (Linnaeus, 1758) (Bufonidae: Anura), en una localidad de Sucre, Colombia. Caldasia 33(2):495-505. 
SANTOS, T.G.; ROSSA-FERES, D.C. \& CASATTI, L. 2007. Diversidade e distribuição espaço-temporal de anuros em região com pronunciada estação seca no sudeste do Brasil. Iheringia, Série Zoológica 97:37-49.

SEEBACHER, F. \& ALFORD, R.A. 1999. Movement and microhabitat use of terrestrial amphibian (Bufo marinus) on a tropical island: seasonal variation and environmental correlates. Journal of Herpetology 33:208-214.

URBINA-C, J. \& LONDOÑO-M, M.C. 2003. Distribución de la comunidad de herpetofauna asociada a cuatro áreas con diferente grado de perturbación en la Isla Gorgona, Pacífico colombiano. Revista de la Academia Colombiana de Ciencias Exactas, Físicas y Naturales 27(102):105-113.

VARGAS-S, F. \& BOLAÑOS-L, M.E. 1999a. Anfibios y reptiles presentes en hábitats perturbados de selva lluviosa tropical en el Bajo Anchicayá, Pacífico colombiano. Revista de la Academia Colombiana de Ciencias Exactas, Físicas y Naturales 23:499-511.

VIEIRA, W.L.; SANTANA, G. \& ARZABE, C. 2009. Diversity of reproductive modes in anurans communities in the Caatinga (dryland) of northeastern Brazil. Biodiversity and Conservation 18(1):55-66.

ZIMMERMAN, B. \& BIERREGAARD, R. 1986. Relevance of the equilibrium theory of island biogeography and species area relations to conservation with a case from Amazonia. Journal of Biogeography 13:133-143 